\title{
Spatial Fuzzy C-Means Clustering based Segmentation of Tumor in Vertebral Column Images
}

\author{
V. Asanambigai \\ Assistant Professor \\ Computer Science and Engineering, \\ Annamalai University \\ India
}

\author{
J. Sasikala \\ Assistant Professor \\ Computer Science and Engineering, \\ Annamalai University \\ India
}

\begin{abstract}
Precise detection and segmentation of tumor that represents uncontrolled growth of tissue, in vertebral column is complex due to vertebrae shapes, gaps in the cortical bone, internal boundaries, as well as the noisy, incomplete or missing information from the MRI or CT scan images, and becomes a challenging task. This paper presents an elegant method using FCM clustering with spatial information for segmenting tumor region of such images. It segments the cluster image corresponding to the largest centroid and uses a few of the morphological operations for removing unwanted regions. It includes the results of three test images for illustrating the goodness of the proposed method.
\end{abstract}

\section{General Terms}

image processing, segmentation

\section{Keywords}

fuzzy c-means clustering, image segmentation.

\section{INTRODUCTION}

The vertebral column plays an important role in the human biomechanical system. The vertebral column extends from the skull to its anchoring point in the pelvis, through which it transfers the weight of the head and trunk to the legs. It also houses and protects the spinal cord. Cartilage pads with jellylike filling called inter-vertebral disks separate the vertebrae3. The posterior body surfaces and arches of the stacked vertebrae form the spinal canal. Tumors in the vertebral column are becoming a common problem affecting many people over the world, as it is the most common site for cancer cells to form metastases, following the lung and the liver cancers. The other type of tumor is the malignant bone tumor, that is quite rare and usually grows slowly either from the bone or disc elements of the spine [1,2].

The most noticeable sign of tumor is pain, which comes from the tumor's presence in the vertebral column, pushing on sensitive nerve endings or causing spinal instability. When the tumor presses on the spinal cord, symptoms may begin with numbness or tingling in the arms or legs. Next, there may be clumsiness, not knowing where the feet are, and difficulty with buttons or keys. As the disease progresses, the symptoms may grow to include weakness, inability to move the legs and eventually paralysis.

Tumors are usually detected by CT or MRI examination yielding different characterization of tissue. Radiologists analyze the medical images, and diagnose a patient's disease by interpreting medical images with their experience, knowledge, and wisdom. The details of the type, precise position and volume of tumors are essential for appropriate treatment with surgical planning. The radiologists require a computer aided diagnostic system (CADS) that has the abilities of learning and pattern recognition, for the purpose of attaining a correct diagnosis. The CADS involves preprocessing, segmentation, feature extraction and classification. Among them, image segmentation is one of the most crucial tasks in medical image analysis, which segments the suspicious tumor region [3].

In recent years, digital images of vertebral column were analyzed, segmented and studied, and many research papers were published [4]. The segmentation methods in the published papers have been tailored with several classes of algorithms, such as contour and edge detection [5], seeded region growing [6] and B-spline active surface [7], which are not suited to an interactive scenario requirement in CADS.

The goal of this paper is to suggest an elegant method for segmenting vertebral column images using fuzzy-c-means (FCS) clustering with spatial information. The paper is organized with four sections containing introduction, proposed method (PM) using spatial FCS (SFCS), results and discussions, and conclusion.

\section{PROPOSED METHOD}

In the PM, the vertebral column image is initially preprocessed through converting the color image into gray image, and filtering of noise and other artifacts. The noises in CT or MRI images are due to thermal effects. Median filter is applied in the proposed method.

FCM is one of the most popular algorithms in fuzzy clustering that permits the member of one fuzzy set being the members of other fuzzy sets of the same image and widely applied to medical mages $[8,9]$. It utilizes a membership function $\mu_{m n}$ to indicate the degree of membership of the $n$-th object to the mth cluster in the range of $(0,1)$. The membership function defines the fuzziness and the information contained in an image. The FCM is sensitive to noises and other artifacts, and results too many small regions in the segmented results. This is because FCM does not consider any spatial information of the given image. Recently, SFCM algorithm to include spatial information of the image has been suggested and its cost function containing spatial information can be written as [10]

$$
\text { Minimize } \Phi=\sum_{m=1}^{C} \sum_{n=1}^{N} \mu_{m n}^{l}\left\|i_{n}-v_{m}\right\|^{2}
$$

Subject to

$$
\sum_{m=1}^{C} \mu_{m n}=1 ; \quad 0 \leq \mu_{m n} \leq 1 ; \quad \sum_{m=1}^{N} \mu_{m n}>0
$$


Table 1 Test Images
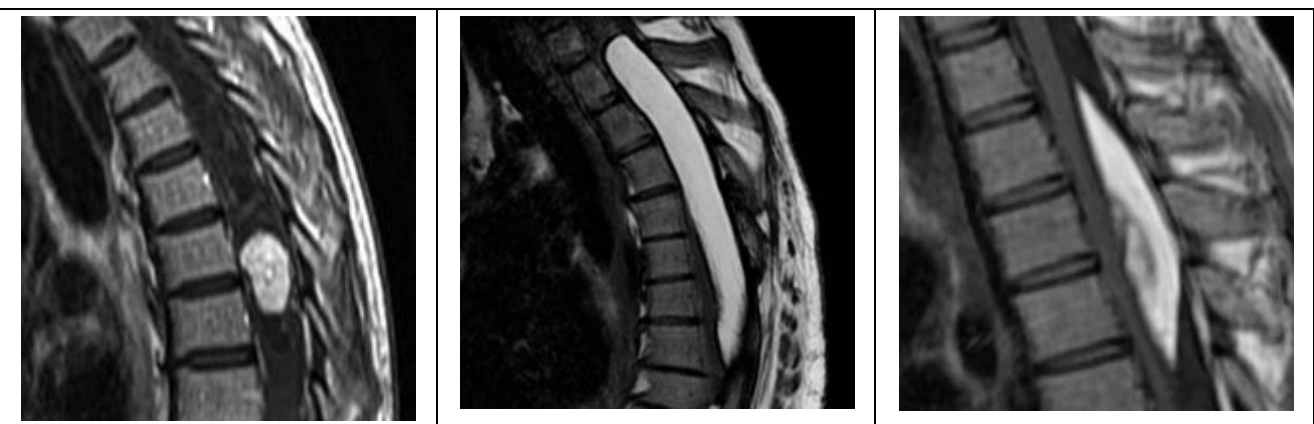

Table 2 Fuzzy Clustered Images



Table 3 Segmented Images
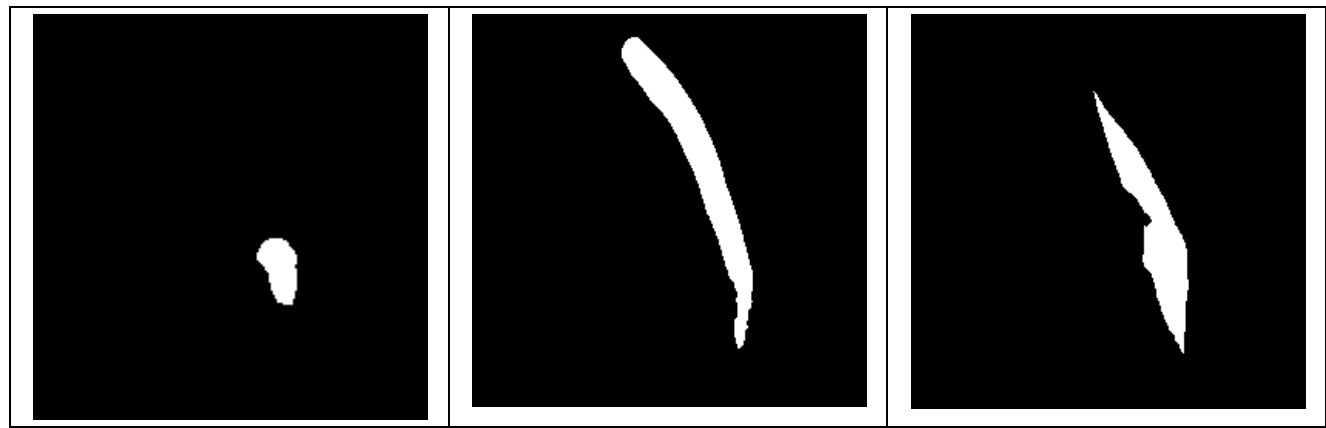

Table 4 Segmented Region Marked on Test Images 


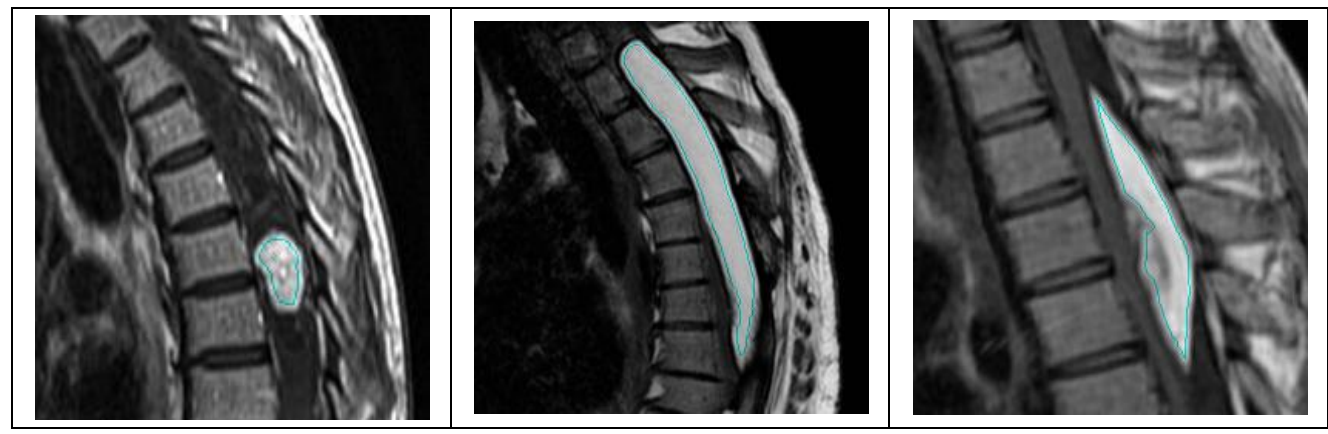

The membership functions $\mu_{m n}$ with spatial information, and the centroids $v_{m}$ are updated by the following equations:

$$
\begin{aligned}
& \mu_{m n}=\frac{a_{m n}^{p} h_{m n}^{q}}{\sum_{k=1}^{C} a_{k n}^{p} h_{k n}^{q}} \\
& v_{m}=\frac{\sum_{n=1}^{N} \mu_{m n}^{l} i_{n}}{\sum_{n=1}^{N} \mu_{m n}^{l}}
\end{aligned}
$$

Where

$$
\begin{aligned}
& a_{m n}=\frac{\left\|i_{n}^{i}-v_{m}\right\|^{-2 /(l-1)}}{\sum_{k=1}^{C}\left\|i_{n}-v_{k}\right\|^{-2 /(l-1)}} \\
& h_{m n}=\sum_{k \in N_{n}} a_{m k}
\end{aligned}
$$

$l(>1)$ is a parameter controlling the fuzziness of the resultant segmentation

$\mathrm{C}$ indicates the number of cluster centroids

$\mathrm{N}$ represents the total number of pixels in the image

The algorithmic steps of the SFCM is outlined below:

1. Initialize $\mu_{m n}$ with random values subject to constraints of Eq. (2).

2. Set initial cluster centroids $v_{m}$ in the range of (0-255).

3. Calculate the value of the cost function $\Phi$ by Eq. (1).

4. Update $\mu_{m n}$ and $v_{m}$ using Eqs. (3) and (4).

5. Check for convergence by comparing the cost function values $\Phi$ of the subsequent iterations. If converged, go to next step; else go to step (3).

6. Each clusters contains the membership values of all the pixels. Defuzzify the clusters by choosing the "winning" MF values. That is, for each pixel, compare the MF values of all the clusters, and choose the cluster with largest MF value as the winning cluster and set its pixel value by the corresponding cluster centroid.

7. Stop
The vertebral column image is segmented into three fuzzy clusters by the above SFCM and the clustered image corresponding to the largest centroid represent the segmented result containing the tumor part. The output consists of tumor region as well as the other regions which have the same luminance and color values as the tumor. The regions which are smaller than the tumor are to be removed by a set of morphological operations. (7) Region growing algorithm can also be used in selecting the tumor part and eliminating the similar unwanted regions. In the proposed work, a set of morphological operations are performed.

\section{RESULTS AND DISCUSSIONS}

The PM was tested on three vertebral column images, presented in Table 1. The number of centroids was chosen as three. The clustered images representing membership corresponding to optimal centroids are presented in Table 2 . The segmented image corresponding to the largest value of the centroids of all the test images are given in Table 3. The segmented portion is marked on the test images and given in Table 4. The inspection of these images indicates that the PM is able to perfectly segment tumor region.

The performances of the PM are evaluated by the following indices [11]:

$$
\begin{gathered}
\% S E I=\left(\frac{t n}{t p+f n}\right) \times 100 \\
\% S P I=\left(\frac{t n}{t n+f p}\right) \times 100 \\
J I=\frac{\Theta_{G T} \cap \Theta_{P M}}{\Theta_{G T} \cup \Theta_{P M}} \\
D C=2 \times \frac{\Theta_{G T} \cap \Theta_{P M}}{\Theta_{G T}+\Theta_{P M}}
\end{gathered}
$$

where

$S E I$ and $S P I$ is the sensitivity and specificity index respectively.

$t p, t n, f p$, and $f n$ indicates true positive, true negative, false positive and false negative respectively.

$J I$ and $D C$ indicates Jaccard index and Dice Coefficient

$\Theta_{G T}$ and $\Theta_{P M}$ represents ground truth region and area detected by the PM respectively. 
Table 5 Performance Indices

\begin{tabular}{|c|c|c|c|}
\hline & Image-1 & Image-2 & Image-3 \\
\hline SEI & 85.37 & 86.12 & 88.03 \\
\hline SPI & 90.11 & 93.21 & 91.16 \\
\hline JI & 0.853 & 0.837 & 0.861 \\
\hline DC & 0.901 & 0.863 & 0.771 \\
\hline
\end{tabular}

These indices are calculated for the three test images under study and given in Table 5. The values of these indices clearly indicate the superior performance of the PM.

\section{CONCLUSIONS}

An elegant method using FCM clustering with spatial information of the images has been suggested in this paper. The method has been applied on vertebral column images for segmenting the tumor region. It defuzzified the membership function values of the optimal clusters for segmenting the clustered images. The segmented result corresponding to the largest centroid represented the tumor region. This segmented image further processed through a set of the morphological operations to eliminate the unwanted similar regions, which have the same luminance and color values of the tumor. The results of three test images clearly illustrated that the PM is able to segment the tumor region effectively.

\section{ACKNOWLEDGMENTS}

The authors gratefully acknowledge the authorities of Annamalai University for the facilities offered to carry out this work.

\section{REFERENCES}

[1] Yao J, O'Connor SD, Summers RM. (2006). Automated spinal column extraction and partitioning. In Proceedings of the 3rd IEEE International Symposium on Biomedical Imaging: Nano to Macro, Arlington, VA, 390-393

[2] Hahn M, Beth T. (2004) Balloon based vertebra separation in CT images. In Proceedings of the 17th IEEE Symposium on Computer-Based Medical Systems, Los Alamitos, CA, 310-315

[3] Ruiz D, Berenguer V, Soriano A, SáNchez B. (2011). A decision support system for the diagnosis of melanoma:
A comparative approach. Expert Systems with Applications, 38(12):217-23.

[4] A. Suzani, A. Rasoulian, S. Fels, R. N. Rohling, and P. Abolmaesumi. (2014). Semi-automatic segmentation of vertebral bodies in volumetric $\mathrm{mr}$ images using a statistical shape-pose model, Proc. SPIE 9036, Medical Imaging, vol. 9036, 1- 6.

[5] T. C. Mann RS, Constantinescu CS. (2007). Upper cervical spinal cord cross-sectional area in relapsing remitting multiple sclerosis: application of a new technique for measuring cross sectional area on magnetic resonance images, J Magn Reson Imaging, 26: 61-67.

[6] C. M. Schmit BD. (2004) Quantification of morphological changes in the spinal cord in chronic spinal cord injury using magnetic resonance imaging, IEEE Eng Med Biol Soc, 6: 4425-4453,.

[7] P. G. B. G. M. D. A. S. Coulon O, Hickman SJ. (2002). Quantification of spinal cord atrophy from magnetic resonance images via a b-spline active surface model. Magn Reson Med, 47: 1176-1185.

[8] W,.Cai, S. Chen, D. Zhang, Fast and robust fuzzy cmeans clustering algorithms incorporating local information for image segmentation, Pattern Recognition 40 (2007) 825-838

[9] W.K.Lei, B.N. Li, M.C.Dong, M.I.VAi. (2007). AFCECG an adaptive fuzzy ECG classifier, in Proceedings of the 11th World Congress on Soft Computing in Industrial Applications (WSC11), Advances in Soft Computing 39: 189-199.

[10] K. S. Chuang, H. L. Hzeng, S. Chen, J. Wu, T. J. Chen. (2006). Fuzzy c-means clustering with spatial information for image segmentation, Computerized medical imaging and graphics, 30: 9-15.

[11] V. Asanambigai, J. Sasikala, Adaptive chemical reaction based spatial fuzzy clustering for level set segmentation of medical images, Ain Shams Engineering Journal, http://dx.doi.org/10.1016/j.asej.2016.08.003. 